\title{
COVERS OF DEHN FILLINGS ON ONCE-PUNCTURED TORUS BUNDLES. II
}

\author{
MARK D. BAKER
}

(Communicated by Frederick R. Cohen)

\begin{abstract}
Let $M$ be a compact, orientable 3-manifold that fibers over $S^{1}$ with fiber a once-punctured torus. We prove that infinitely many Dehn fillings on $M$ yield manifolds with virtually $\mathbb{Z}$-representable fundamental groups.
\end{abstract}

\section{INTRODUCTION}

Let $M$ be a compact, orientable 3-manifold that fibers over the circle with fiber a once-punctured torus. In this paper we use cut and paste techniques to prove that infinitely many Dehn fillings on $M$ yield manifolds with virtually $\mathbb{Z}$-representable fundamental groups. This extends the results in [B].

This work was motivated by the question of whether every compact, orientable, irreducible 3-manifold with infinite fundamental group is finitely covered by a Haken manifold (is virtually Haken). It follows from work of Thurston, Waldhausen, and others that an affirmative answer would have important consequences for the classification of 3-manifolds with infinite fundamental group.

A group is virtually $\mathbb{Z}$-representable if it has a finite index subgroup with a nontrivial representation to the integers. If $N$ is a compact 3-manifold, the virtual $\mathbb{Z}$-representability of $\pi_{1}(N)$ is equivalent to the existence of a finite cover $\tilde{N} \rightarrow N$ with $\operatorname{rank} H_{1}(\tilde{N}) \geq 1$. If $N$ is also orientable and irreducible, it follows that $\tilde{N}$ is Haken.

The manifolds obtained by Dehn filling on once-punctured torus bundles have been much studied (see [FH], [CJR], [T]). In particular they provided the first examples of hyperbolic (hence irreducible) non-Haken 3-manifolds.

\section{STATEMENT OF RESUlts}

Let $M$ be a compact, orientable 3-manifold that fibers over $S^{1}$ with fiber a once-punctured torus, $T_{0}$, and characteristic homeomorphism $h: T_{0} \rightarrow T_{0}$ which is the identity on $\partial T_{0}$, the boundary of $T_{0}$. Then

$$
M \cong T_{0} \times[0,1] /(h(x), 0) \sim(x, 1),
$$

Received by the editors June 16, 1989.

1980 Mathematics Subject Classification (1985 Revision). Primary 57M10, 57N10. 
where we identify $T_{0} \times\{1\}$ to $T_{0} \times\{0\}$ by $h$.

Choose a point $b$ in $\partial T_{0}$ and consider the loops $\alpha=b \times[0,1] / \sim$ and $\beta=\partial T_{0}$ (see Figure 1).

Denote by $M(\mu, \lambda)$ the manifold obtained from Dehn filling on $M$ with respect to the loop $\alpha^{\mu} \beta^{\lambda}$ in $\partial M$. By Dehn filling on a 3-manifold $N$ with respect to a simple loop in a boundary torus we mean gluing a solid torus to $\partial N$ so that this loop bounds a meridional disk in the solid torus.

Let $D_{x}$ denote the right-handed Dehn twist about the loop $x$ and $D_{y}$ denote the left-handed Dehn twist about the loop $y$ in $T_{0}$. Then any orientation preserving homeomorphism $h: T_{0} \rightarrow T_{0}$ can be represented up to isotopy by a composition of powers of $D_{x}$ and $D_{y}$. We prove:

Theorem 1. Let $M$ be a once-punctured torus bundle over $S^{1}$ with characteristic homeomorphism of the form $D_{x}^{r_{1}} \circ D_{y}^{s_{1}} \circ \cdots \circ D_{x}^{r_{k}} \circ D_{y}^{s_{k}}$ such that $4 \mid s_{i}$ for each $i=1, \ldots, k$. Let $(\mu, \lambda)$ be relatively prime integers with $|\lambda| \geq 2$, and let $R=r_{1}+\cdots+r_{k}$ and $m=|R \mu+2 \lambda|$. Then $M(\mu, \lambda)$ has virtually $\mathbb{Z}$-representable fundamental group provided there is a prime $p \mid \lambda$ such that one of the following conditions on $m$ and $p$ holds:

(a) $p=2$ and $4 \mid m$,

(b) $p \geq 3$ and $p \mid m$,

(c) $p \nmid m, p<m$, and $m \geq 4$.

Remarks. 1. Since $M(\mu, \lambda)=M(-\mu,-\lambda)$, we will assume that $\mu \geq 1$. For a given $\mu \geq 1, M(\mu, \lambda)$ has virtually $\mathbb{Z}$-representable fundamental group for all but finitely many $\lambda$.

2. We assume that g.c.d. $\left(r_{1}, \ldots, r_{k}\right)=1$ since the complementary case was treated in [B]. Theorem 1 holds true if $4 \mid r_{i}$ for each $i=1, \ldots, k$ and g.c.d. $\left(s_{1}, \ldots, s_{k}\right)=1$, provided we let $S=s_{1}+\cdots+s_{k}$ and $m=|S \mu+2 \lambda|$.

We have the following consequence of Theorem 1:

Theorem 2. Let $M$ be a once-punctured torus bundle over $S^{1}$. Then infinitely many Dehn fillings on $M$ yield manifolds with virtually $\mathbb{Z}$-representable fundamental groups. Specifically, there exists an integer $c \in\{1,2,3,4,6\}$, depending on $M$, such that for each $\mu$ a nonzero multiple of $c, M(\mu, \lambda)$ has virtually $\mathbb{Z}$-representable fundamental group for all but finitely many of the $\lambda$ coprime to $\mu$.

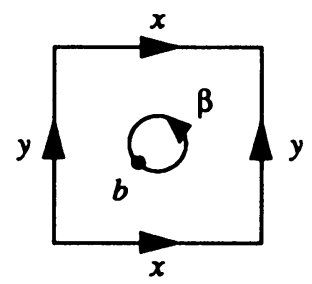

FIGURE 1 
In $\S 3$ we prove Theorem 1 . Section 4 is devoted to showing how Theorem 2 follows from Theorem 1 .

\section{Proof of Theorem 1}

For a torus bundle $M$, with characteristic homeomorphism $h$, and Dehn filling parameters $(\mu, \lambda)$ as in Theorem 1, we prove our result by constructing a finite cover $\widetilde{M} \rightarrow M$ such that:

(i) The cover $\widetilde{M} \rightarrow M$ extends to a cover $N \rightarrow M(\mu, \lambda)$ by Dehn filling on $\widetilde{M}$ and on $M$.

(ii) $\operatorname{rank} H_{1}(\widetilde{M})>$ number of components of $\partial \widetilde{M}$.

Property (ii) guarantees that any manifold obtained by Dehn filling on $\widetilde{M}$ (hence $N$ ) has positive first Betti number; thus $M(\mu, \lambda)$ has virtually $\mathbb{Z}$ representable fundamental group.

The cover $\widetilde{M}$ is obtained by constructing a cover $F \stackrel{\pi}{\rightarrow} T_{0}$ which $h$ lifts to a homeomorphism $\tilde{h}: F \rightarrow F$. The mapping torus, $\widetilde{M}$, of the pair $(F, \tilde{h})$ is the desired cover of $M$.

3.1. Recall that the characteristic homeomorphism, $h$, of $M$ is of the form $D_{x}^{r_{1}} \circ D_{y}^{s_{1}} \circ \cdots \circ D_{x}^{r_{k}} \circ D_{y}^{s_{k}}$ where $4 \mid s_{i}$ for $i=1, \ldots, k$.

For each integer $d \geq 3$, we construct a $4 d$-fold cover $S \stackrel{\pi}{\rightarrow} T_{0}$ corresponding to the kernel of the map

$$
\theta: \pi_{1}\left(T_{0}\right) \rightarrow \mathbb{Z} / d \oplus \mathbb{Z} / 4
$$

defined by $\theta([x])=(1,0)$ and $\theta([y])=(0,1)$. The loop $x$ (resp. $y$ ) in $T_{0}$ is covered by four loops $\tilde{x}_{1}, \ldots, \tilde{x}_{4}$ (resp. $d$ loops $\tilde{y}_{1}, \ldots, \tilde{y}_{d}$ ) in $S$ that project $d$ to 1 onto $x$ (resp. 4 to 1 onto $y$ ). The surface $S$ for $d=5$ is pictured in Figure 2.

3.2. We now alter $S$ by cutting and pasting to obtain a cover $F \stackrel{\pi}{\rightarrow} T_{0}$ to which both Dehn twists $D_{x}$ and $D_{y}^{4}$ lift.

It will be helpful to think of $S$ as being divided into a grid by the curves $\left\{\tilde{x}_{i}\right\},\left\{\tilde{y}_{i}\right\}$. Thus $S$ consists of 4 rows numbered from bottom to top. Each row contains $d$ squares, each with a boundary circle (see Figure 2).

Make $2 d$ vertical cuts in $S$, one between each circle of row 1 (resp. row 3 ) and the circle directly above in row 2 (resp. row 4 ). Now identify the left edge of each cut to the right edge of the cut $d-2$ to the right $(\bmod d)$. Call the resulting surface $F$. The surface $F$ for $d=5$, with identifications numbered, is shown in Figure 3.

Lemma 3.1. The surface $F$ is a $4 d$-fold cover of $T_{0}$. If $d$ is odd, $F$ has genus $2 d-1$ and four boundary circles, each of which projects $d$ to 1 onto $\beta=\partial T_{0}$. If $d$ is even, $F$ has genus $2 d-3$ and eight boundary circles, each projecting $d / 2$ to 1 onto $\beta$.

The loop $x$ (resp. $y$ ) in $T_{0}$ is covered by four loops $\tilde{x}_{1}, \ldots, \tilde{x}_{4}$ (resp. $d$ loops $\tilde{y}_{1}, \ldots, \tilde{y}_{d}$ ) in $F$ that project $d$ to 1 onto $x$ (resp. 4 to 1 onto $y$ ). $F$ 


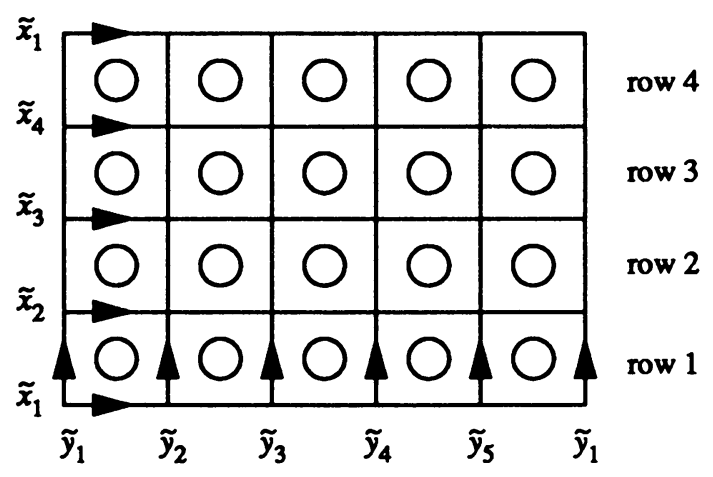

FIGURE 2

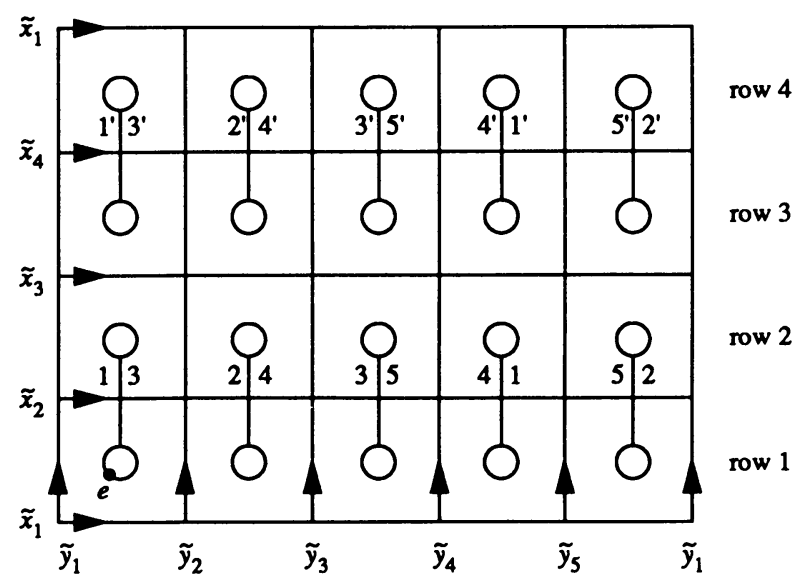

FIGURE 3

is divided into a grid by the curves $\left\{\tilde{x}_{i}\right\},\left\{\tilde{y}_{i}\right\}$. We number the four rows of $F$ from bottom to top (see Figure 3).

Since the loops $\left\{\tilde{y}_{i}\right\}$ in $F$ project 4 to 1 onto $y$ in $T_{0}$, the Dehn twist $D_{y}^{4}$ lifts to simultaneous Dehn twists about the $\left\{\tilde{y}_{i}\right\}$.

Moreover, $D_{x}$ lifts to a homeomorphism, $\widetilde{D}_{x}$, of $F$ which can be viewed as a $1 / d$ "fractional" Dehn twist about each of the $\left\{\tilde{x}_{i}\right\}$. Indeed, if we require our lifts to fix the basepoint, $e$ of $F$ in the first square of row 1 (see Figure 3), then $\widetilde{D}_{x}$ fixes pointwise rows 1 and 3 while shifting rows 2 and 4 each $d-1$ squares to the right $(\bmod d)$.

Since $D_{x}$ and $D_{y}^{4}$ both lift to $F$, it follows that any homeomorphism $h: T_{0} \rightarrow T_{0}$ of the form $D_{x}^{r_{1}} \circ D_{y}^{s_{1}} \circ \cdots \circ D_{x}^{r_{k}} \circ D_{y}^{s_{k}}$ where $4 \mid s_{i}$ also lifts to a homeomorphism $\tilde{h}: F \rightarrow F$. Denote by $\widetilde{M}$ the mapping torus of $(F, \tilde{h})$. 
3.3. We prove that $H_{1}(\widetilde{M})$ satisfies property (ii) that was given at the beginning of $\S 3$ :

Lemma 3.2. $\operatorname{rank} H_{1}(\widetilde{M})>$ number of components of $\partial \widetilde{M}$.

Proof. This is equivalent to the existence of non-boundary classes in $H_{1}(F)$ that are fixed by $\tilde{h}_{*}$ (see $[\mathrm{B}]$ or $[\mathrm{H}]$ ).

A portion of $F$ is shown in Figure 4 (see p. 1104). The class $[\gamma]+[\delta]$ in $H_{1}(F)$ corresponding to the loops $\gamma, \delta$ is fixed by $\tilde{h}_{*}$ since $\widetilde{D}_{x}$ fixes $\gamma$ and $\delta$ and they each intersect with opposite orientations the same two Dehn twist curves in the set $\left\{\tilde{y}_{i}\right\}$.

Finally $[\gamma]+[\delta]$ is not homologous in $H_{1}(F)$ to a sum of boundary circles in $F$, since $\gamma$ and $\delta$ have nonzero intersection with the curves $\sigma$ and $\rho$ while all the boundary circles have null intersection with $\sigma$ and $\rho$.

Remark. This argument generalizes to show that $\operatorname{rank} H_{1}(\widetilde{M}) \geq$ (number of components of $\partial \widetilde{M})+[d / 3]$.

3.4. Now consider $M$ with characteristic homeomorphism $h$ as in Theorem 1. We use the covers $\widetilde{M} \rightarrow M$ constructed in 3.2 to prove our result in the case where the integers $m, p$ in the theorem satisfy condition (a) or (b).

Let $(\mu, \lambda)$ be Dehn filling parameters for $M, \mu \geq 1,|\lambda| \geq 2$. Recall that $R=r_{1}+\cdots+r_{k}, m=|R \mu+2 \lambda|$, and suppose $p \geq 3$ is a prime factor of $\lambda$ dividing $m$ (condition (b)).

Let $\widetilde{M}$ be the mapping torus of $(F, \tilde{h})$ for $F$ corresponding to $d=p$. Given Lemma 3.2, all that remains to show is that the loop $\alpha^{\mu} \beta^{\lambda}$ in $\partial M$ lifts to loops in $\partial \widetilde{M}$. Since $p$ divides $m$ and $\lambda, p$ also divides $R$ and it follows from the construction of $F$ and the action of $\widetilde{D}_{x}$ on $F$ that $\tilde{h}$ fixes pointwise the four boundary components of $F$, each of which projects $p$ to 1 to $\beta=\partial T_{0}$. Thus $\alpha, \beta^{p}$ and hence $\beta^{\lambda}$ in $\partial M$ lift to loops in $\partial \widetilde{M}$ so that $\alpha^{\mu} \beta^{\lambda}$ lifts to loops in $\partial \widetilde{M}$. Choose one lift in each component of $\partial \widetilde{M}$ and label them $\left\{c_{i}\right\}$.

Lemma 3.3. The cover $\widetilde{M} \rightarrow M$ extends to a cover $N \rightarrow M(\mu, \lambda)$ by Dehn filling on $\widetilde{M}$ and $M$ with respect to $\left\{c_{i}\right\}$ in $\partial \widetilde{M}$ and $\alpha^{\mu} \beta^{\lambda}$ in $\partial M$.

Lemma 3.4. $\operatorname{rank} H_{1}(N) \geq 1$.

Proof. Since $\operatorname{rank} H_{1}(\widetilde{M})>$ number of components of $\partial \widetilde{M}$ (Lemma 3.2), it follows that all manifolds obtained by Dehn filling on $\widetilde{M}$ (hence $N$ ) have positive first Betti number.

If $p=2$ and $4 \mid m$ (condition (a)), then $4 \mid R$ and we let $\widetilde{M}$ be the mapping torus of $(F, \tilde{h})$ for $F$ corresponding to $d=4$. Now the above argument applies almost verbatim.

3.5. In this section we conclude the proof of Theorem 1 with the case where $M, h$, and $(\mu, \lambda)$ are such that the integers $m$ and $p$ satisfy condition (c): $p \nmid m, p<m$, and $m \geq 4$. 


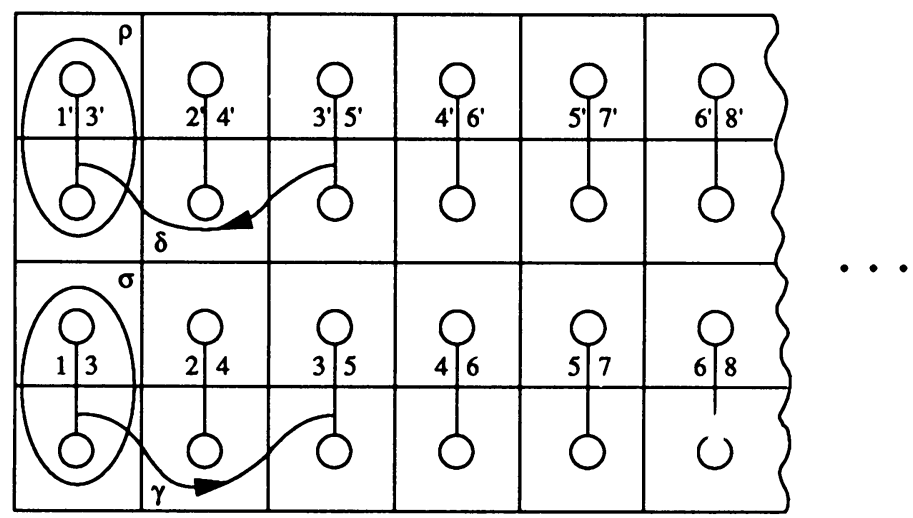

FIGURE 4

For clarity of exposition we will assume that $m$ is odd. The proof for $m$ even is analogous.

Consider the cover $\widetilde{M} \rightarrow M$ where $\widetilde{M}$ is the mapping torus of $(F, \tilde{h})$ for $F$ corresponding to $d=m$ (see $\S 3.2$ ). By Lemma 3.2, all Dehn fillings on $\widetilde{M}$ yield manifolds with positive first Betti number.

We now show that the loop $\alpha^{\mu} \beta^{\lambda}$ in $\partial M$ lifts to loops in two of the four boundary tori of $\widetilde{M}$, which we denote by $T_{i}$ and index so that $\tilde{\beta}_{i}=T_{i} \cap F$ is the circle in the $i$ th row of $F$ for $i=1, \ldots, 4$. The circles $\tilde{\beta}_{i}$ of $F$ project $m$ to 1 onto $\beta=\partial T_{0}$ (see $\S 3.2$ ).

Here it is important to specify orientations. All bundles are constructed by identifying the back face, $F \times\{1\}$, to the front face, $F \times\{0\}$, via $h$ $((h(x), 0) \sim(x, 1))$. All curves transverse to $F$ are oriented from front to back and all boundary circles of $F$ are given a counterclockwise orientation.

Lemma 3.5. The loop $\alpha^{\mu} \beta^{\lambda}$ in $\partial M$ lifts to loops in $T_{2}$ and $T_{4}$ of $\partial \widetilde{M}$.

Proof. First note that $\tilde{h}$ shifts rows 2 and 4 of $F$, rotating $\tilde{\beta}_{2}$ and $\tilde{\beta}_{4}$ (recall action of $\left.\widetilde{D}_{x}, \S 3.2\right)$. One checks that the loops $\alpha \beta^{R(m-1) / 2}$ and $\beta^{m}$ lift to loops in $T_{2}$ and $T_{4}$. But then $\alpha^{\mu} \beta^{\lambda}$ also lifts to a loop, being a combination of $\alpha \beta^{R(m-1) / 2}$ and $\beta^{m}$. Indeed, $m=|R \mu+2 \lambda| \Rightarrow R \mu+2 \lambda \equiv 0(\bmod m) \Rightarrow$ $\mu R(m-1)-2 \lambda \equiv 0(\bmod m) \Rightarrow \mu R(m-1) / 2 \equiv \lambda(\bmod m)$.

We would be done except for the fact that $\alpha^{\mu} \beta^{\lambda}$ does not lift to loops in $T_{1}$, and $T_{3}$ of $\widetilde{M}$. We resolve this problem by altering $\widetilde{M}$ to obtain a new cover $M^{\prime} \rightarrow M$ that satisfies Lemma 3.2 , and has the property that $\alpha^{\mu} \beta^{\lambda}$ lifts to loops in each component of $\partial M^{\prime} . M^{\prime}$ is the mapping torus of $\left(F^{\prime}, h^{\prime}\right)$ where $F^{\prime}$ is obtained from $F$ by cut and paste alteration of the boundary circles $\tilde{\beta}_{1}$, $\tilde{\beta}_{3}$ in rows 1 and 3 . 
The pattern of cut and paste depends on the parity of $\lambda$. In each case we first illustrate the general procedure with an example. Consider $M$ with homeomorphism $h=D_{x} \circ D_{y}^{4}$ and $(\mu, \lambda)=(1,-4)$. Then $R=1, m=7$, and $p=2$. Starting with $F \stackrel{\pi}{\rightarrow} T_{0}$ for $d=m=7$, we obtain $F^{\prime} \stackrel{\pi}{\rightarrow} T_{0}$ by making eight horizontal cuts and identifications in $F$ as pictured in Figure 5. Rows 1 and 3 of $F^{\prime}$ each contain two boundary circles that project 2 to 1 onto $\beta$ and three circles that project 1 to 1 onto $\beta$. It is clear from the construction of $F^{\prime}$ that $D_{x}$ and $D_{y}^{4}$ lift to $F^{\prime}$ and hence that $h$ lifts to a homeomorphism $h^{\prime}: F^{\prime} \rightarrow F^{\prime}$. The loop $\alpha \beta^{-4}$ in $\partial M$ lifts to loops in the boundary tori of $M^{\prime}$ so that $M^{\prime} \rightarrow M$ extends to a cover $N^{\prime} \rightarrow M(1,-4)$. The class $[\gamma]+[\delta]$ in $H_{1}\left(F^{\prime}\right)$ corresponding to the curves $\gamma, \delta$ in $F^{\prime}$ that are in Figure 5 is nonboundary and fixed by $h_{*}^{\prime}$. Hence $M^{\prime}$ satisfies Lemma 3.2 and rank $H_{1}\left(N^{\prime}\right) \geq 1$.

In general if $\lambda$ is even, let $p=2$ and construct $\left(F^{\prime}, h^{\prime}\right)$ from $(F, h)$ by making $2 m-6$ horizontal cuts in $F: m-3$ cuts each in rows 1 and 3 between the adjacent boundary circles in the last $m-2$ squares. In each row number the bottom edges of the $m-3$ cuts from left to right and the top edges from right to left and then identify edges accordingly (see Figure 5). Rows 1 and 3 of $F^{\prime}$ each contain two boundary circles that project 2 to 1 onto $\beta$ and $m-4$ boundary circles that project 1 to 1 onto $\beta$.

By construction, $h$ lifts to a homeomorphism $h^{\prime}: F^{\prime} \rightarrow F^{\prime}$, the curves $\gamma, \delta$ in $F$ carry over to $F^{\prime}$ giving a nonboundary class $[\gamma]+[\delta]$ in $H_{1}\left(F^{\prime}\right)$ that is fixed by $h_{*}^{\prime}$, and the loop $\alpha^{\mu} \beta^{\lambda}$ in $\partial M$ lifts to loops in $\partial M^{\prime}$. Thus $M^{\prime} \rightarrow M$ extends to a cover $N^{\prime} \rightarrow M(\mu, \lambda)$ with rank $H_{1}\left(N^{\prime}\right) \geq 1$.

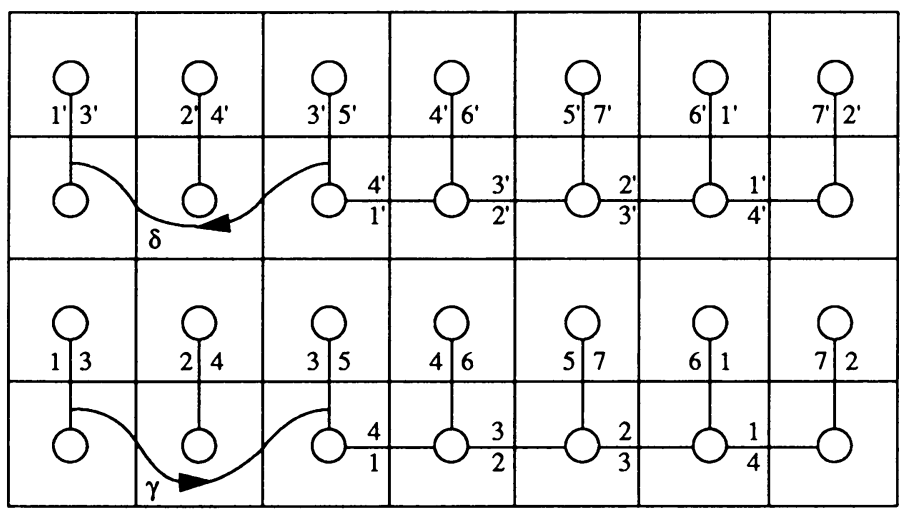

Figure 5 


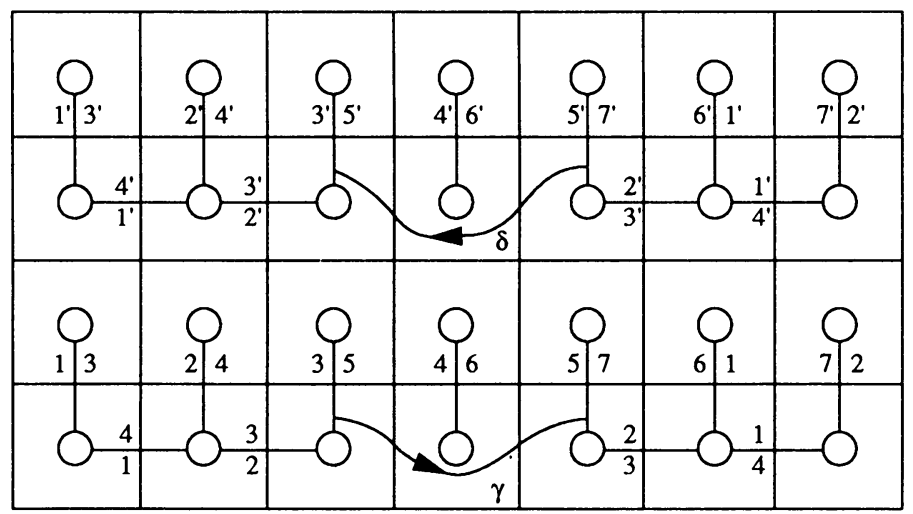

Figure 6

We illustrate the case $\lambda$ odd by $M$ with $h=D_{x} \circ D_{y}^{4}$ and $(\mu, \lambda)=(1,3)$. Hence $R=1, m=7$, and $p=3$. The desired $F^{\prime}$ is pictured in Figure 6 . Note the position of the eight cuts as well as the curves $\gamma, \delta$.

In general if $\lambda$ is odd, then $p$ is odd and $m-p$ is even. We construct $F^{\prime}$ by making $2(m-p)$ horizontal cuts: $m-p$ cuts each in rows 1 and 3 between adjacent circles in all but the $p-2$ middle squares. Now number the bottom edges of the $m-p$ cuts from left to right and the top edges from right to left and identify. Rows 1 and 3 of $F^{\prime}$ each contain $m-p$ boundary circles that project 1 to 1 onto $\beta$ and one circle that projects $p$ to 1 onto $\beta$. The curves $\gamma, \delta$ are in the center of rows 1 and 3 as in Figure 6.

Remark. If $m$ is even, the arguments are similar. The surface $F^{\prime}$ for $m=8$, $p=2$ is illustrated in Figure 7 and for $m=8, p=3$ in Figure 8. Generalizations are left to the reader.

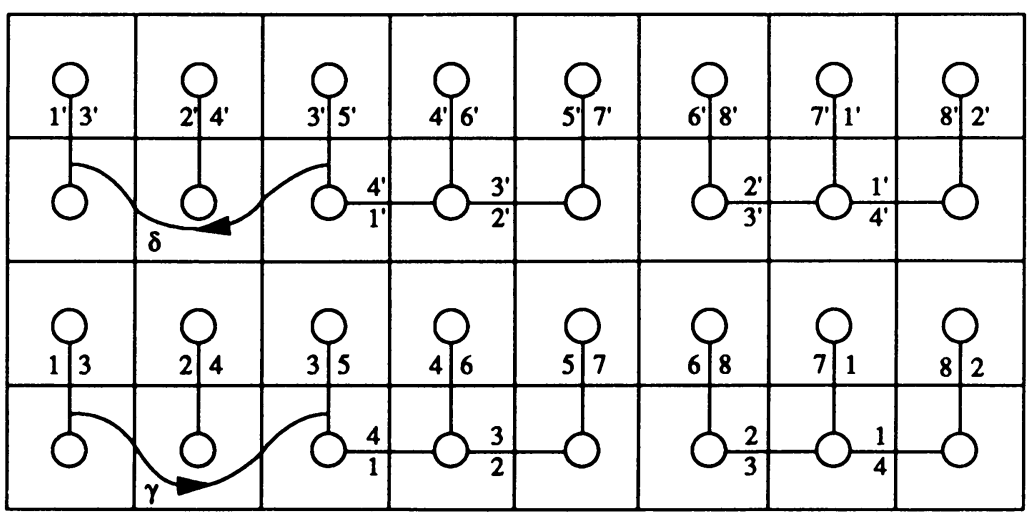

Figure 7 


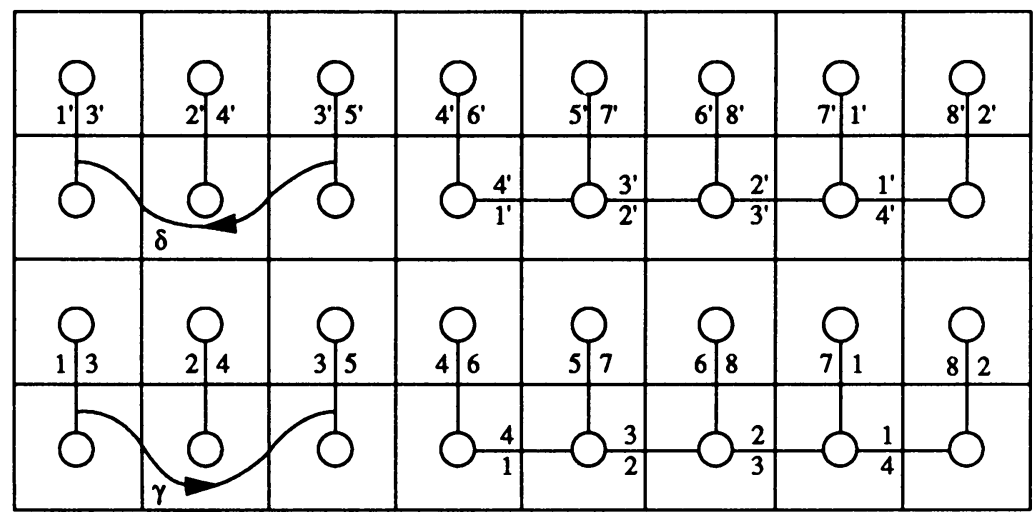

FIGURE 8

\section{Proof of Theorem 2}

Let $M$ be an oriented once-punctured torus bundle over $S^{1}$ with characteristic homeomorphism $h$, and let $[h] \in \mathrm{SL}_{2}(\mathbb{Z})$ be the monodromy matrix representing the action of $h_{*}$ on $H_{1}\left(T_{0}\right)$.

Fact 4.1. $M$ is determined up to oriented $S^{1}$ bundle equivalence by the conjugacy class of $[h]$ in $\mathrm{SL}_{2}(\mathbb{Z})$ (see $[\mathrm{CJR}]$ ).

In particular $\left[D_{x}\right]=\left(\begin{array}{ll}1 & 1 \\ 0 & 1\end{array}\right)$ and $\left[D_{y}\right]=\left(\begin{array}{ll}1 & 0 \\ 1 & 1\end{array}\right)$ generate $\mathrm{SL}_{2}(\mathbb{Z})$.

Let $\Gamma \subset \mathrm{SL}_{2}(\mathbb{Z})$ denote the subgroup generated by $\left(\begin{array}{ll}1 & 1 \\ 0 & 1\end{array}\right)$ and $\left(\begin{array}{ll}1 & 0 \\ 4 & 1\end{array}\right)$. Then Theorem 1 applies to bundles with monodromy $[h] \in \Gamma$.

Fact 4.2. $\Gamma$ contains $\Gamma(4)$ the 4-congruence subgroup, and $\mathrm{SL}_{2}(\mathbb{Z}) / \Gamma(4) \cong$ $\mathrm{SL}_{2}(\mathbb{Z} / 4)$ consists of elements of order $c \in\{1,2,3,4,6\}$.

Now given $M$ with monodromy [h], by Fact 4.2 there exists a smallest positive integer $c \in\{1,2,3,4,6\}$ for which the $c$-fold cyclic cover, $M^{\prime}$, of $M$ has monodromy $\left[h^{c}\right]$ conjugate to an element of $\Gamma$, and by Fact 4.1, $M^{\prime}$ is bundle equivalent to an $M^{\prime \prime}$ to which Theorem 1 applies.

Note that $M^{\prime \prime} \rightarrow M$ is a $c$-fold cover and let $\alpha^{\prime \prime}, \beta^{\prime \prime}$ (resp. $\alpha, \beta$ ) be the basis for $H_{1}\left(\partial M^{\prime \prime}\right)$ (resp. $H_{1}(\partial M)$ ) described in $\S 2$. It is clear that $\alpha^{\prime \prime}$ projects to a loop of the form $\alpha^{c} \beta^{t}$ and $\beta^{\prime \prime}$ projects to $\beta$. Thus Theorem 1 applied to $M^{\prime \prime}$ guarantees that for each $\mu$ a nonzero multiple of $c, M(\mu, \lambda)$ has virtually $\mathbb{Z}$-representable fundamental group for all but finitely many of the $\lambda$ coprime to $\mu$.

\section{REFERENCES}

[B] M. Baker, Covers of Dehn fillings on once-punctured torus bundles, Proc. Amer. Math. Soc. 105 (1989), 747-754.

[CJR] M. Culler, W. Jaco, and H. Rubinstein, Incompressible surfaces in once-punctured torus bundles, Proc. London Math. Soc. (3) 45 (1982), 385-419. 
[FH] W. Floyd and A. Hatcher, Incompressible surfaces in punctured torus bundles, Topology Appl. 13 (1982), 263-282.

[H] J. Hempel, Coverings of Dehn fillings of surface bundles, Topology Appl. 24 (1986), 157-170.

[T] W. Thurston, The geometry and topology of 3-manifolds, xeroxed notes, Princeton University, Princeton, NJ, 1978.

Department of Mathematics, Vanderbilt University, Nashville, Tennessee 37235 